\title{
Sincronia na reprodução de Moenkhausia sanctaefilomenae (Steindachner) (Characiformes: Characidae) na planície de inundação do rio Cuiabá, Pantanal Mato-grossense, Brasil
}

\author{
Luzia da S. Lourenço '; Lúcia A. Mateus ' \& Nadja G. Machado ${ }^{2}$ \\ ${ }^{1}$ Laboratório de Ecologia e Manejo de Recursos Pesqueiros, Instituto de Biociências, Universidade Federal de Mato Grosso. \\ 78060-900 Cuiabá, Mato Grosso, Brasil.E-mail: bioluzia@yahoo.com.br; Imateus@ufmt.br \\ 2 Unidade de Ensino de Tangará da Serra, Secretaria de Estado de Ciência e Tecnologia de Mato Grosso. 78300-000 \\ Tangará da Serra, Mato Grosso, Brasil. E-mail: ngm@terra.com.br
}

\begin{abstract}
Synchrony in the reproduction of Moenkhausia sanctaefilomenae (Steindachner) (Characiformes: Characidae) in the Cuiabá river floodplain, Pantanal of Mato Grosso, Brazil. The reproductive ecology of Moenkhausia sanctaefilomenae (Steindachner, 1907) was studied at 15 lakes of the Cuiabá river floodplain, Mato Grosso. The sex ratio, the timing of reproduction, spawning type and the length of first gonadal maturation were analyzed. We sampled 212 individuals (108 females and 104 males) from June of 2005 to March of 2006. There was not significant difference in the sex ratio, considering the whole period sampled. The biggest frequency of matured females was found in December in all of the lakes. The mean width of the ovarian follicles varied among the periods in December it was larger than others. What it suggests that the spawning happened, probably, among the end of December or beginning of January. That corresponds to the high water and flood in the area. The frequency distribution of the width of the ovarian follicles showed a polimodal pattern. That suggest parceled out spawning, but restricted to the period of December to March. The mean length of gonadal maturation was estimated in $33.9 \mathrm{~mm}$. There was a positive relationship between length of first maturation and the asymptotic length. In synthesis, the data indicate that the reproduction happens in a synchronous way in all of the lakes sampled.
\end{abstract}

KEY WORDS. Gonadal maturation; spawning; sex ratio.

RESUMO. A ecologia reprodutiva de Moenkhausia sanctaefilomenae (Steindachner, 1907) foi estudada em 15 lagoas da planície de inundação do rio Cuiabá, Mato Grosso. Foram analisadas a proporção sexual, a época de maturação, tipo de desova e o comprimento de primeira maturação. Foram amostrados 212 indivíduos (108 fêmeas e 104 machos) no período de junho de 2005 a março de 2006. Não houve diferença significativa na proporção sexual, considerando todo o período amostrado. A maior freqüência de fêmeas maturas foi encontrada em dezembro em todas as lagoas. A largura média dos folículos ovarianos variou entre os períodos, sendo que em dezembro estes foram em média maiores. Sugerindo que a desova ocorreu, provavelmente, entre o final de dezembro e início de janeiro, o que corresponde aos períodos de enchente e cheia na região. A observação de um padrão polimodal da distribuição de freqüência de largura dos folículos ovarianos sugere desova parcelada, mas restrita ao período de dezembro a março. O comprimento médio de maturação foi estimado em $33,9 \mathrm{~mm}$. O comprimento de primeira maturação relaciona-se positivamente com o comprimento assintótico. Em síntese, os dados indicam que a reprodução ocorre de forma sincrônica em todas as lagoas amostradas.

PALAVRAS-CHAVE. Desova; maturação gonadal; proporção sexual.

A reprodução contribui para a manutenção das populações e seu estudo, consequentemente, contribui para o estabelecimento de normas de conservação e manejo das espécies. A proporção sexual é um importante aspecto para ser avaliado na ecologia reprodutiva por ser um indicativo de crescimento da população (RAPOso \& Gurgel 2001), ao fornecer seu potencial reprodutivo (Vicentini \& ARAúJo 2003). Esta pode variar em fun- ção de muitos fatores que atuam de maneira diferente em cada um dos sexos. As principais causas da mortalidade diferencial dos sexos no tempo e no espaço são a predação (RodD \& REZNICK 1997), as diferenças de temperatura (BOHLENA \& RitTeRbusCH 2000), e a mortalidade específica de tamanho (GARCIA et al. 2004).

O comprimento assintótico e o comprimento de primeira maturação são parâmetros de história de vida que co-variam 
(Lowe-McConnell 1999), e dependem da taxa de crescimento individual, que por sua vez sofre efeito das condições do habitat. Assim, ocorre um ajustamento evolutivo destes parâmetros de história de vida em resposta as condições ambientais (Roff 1984), ou seja, o comprimento máximo teórico alcançado pelos indivíduos entre diferentes espécie e populações pode variar dependendo do habitat que ocupa, alterando assim o comprimento de primeira maturação (TARKAN 2006).

O comprimento de primeira maturação, a época de maturação e tipo de desova são traços reprodutivos moldados pelo ambiente que determinam a sobrevivência das populações (Winemiller \& DONALd 1989). Sendo assim, as áreas periodicamente alagadas com mudanças sazonais nas características físico-químicas do ambiente exigem adaptações destes traços para que a entrada de novos indivíduos nas populações seja assegurada (Kramer 1978, VAzZoller 1996, Wootton 1998, LOWE-MCCONNell 1999, Winemiller 2005).

A disponibilidade de recurso (KRAMER 1978, WoOtTON 1998, Winemiller 2005), o tipo de habitat (Veregue \& Orsi 2003), a precipitação (Silvano et al. 2003, Gurgel 2004, Andrade \& Braga 2005), assim como, as características biológicas intrínsecas do organismo como comprimento corporal e filogenia (Alkins-Koo 2000) são alguns dos fatores que determinam a época e a duração do evento reprodutivo das populações. No entanto, o regime de chuvas é o fator que mais influencia os eventos reprodutivos de muitas espécies. Provavelmente, isto ocorre porque o período de expansão do habitat favorece uma maior disponibilidade de recursos (Lowe-McConnell 1999, Mazzoni \& Iglesias-Rio 2002).

Moenkhausia sanctaefilomenae (Steindachner, 1907), conhecida popularmente como olho-de-fogo, pertence a família Characidae, um grupo dominante entre os peixes de água doce da América do Sul (BRitski et al. 1999). A espécie habita rios, riachos e lagoas das planícies de inundação dos rios Parnaíba, São Francisco, Alto Paraná, bacia do rio Paraguai e Uruguai (ReIs et al. 2003). Os indivíduos podem alcançar um comprimento máximo de $70 \mathrm{~mm}$ e são encontrados em áreas com extensa vegetação subemergente e emergente. São ágeis nadadores e ocupam da meia água a superfície para coletar itens alimentares (Machado-Allison 1987, Casatti et al. 2001).

Na planície de inundação do alto rio Paraná, Lizama \& Ambrósio $(1999,2002)$ analisaram a estrutura populacional e o fator de condição de M. sanctaefilomenae, mas estudos sobre a ecologia reprodutiva inexistem. Assim, o presente estudo teve como objetivo comparar o comprimento médio entre machos e fêmeas, analisar a proporção sexual e o comprimento de primeira maturação das fêmeas de M. sanctaefilomenae. Adicionalmente, avaliou-se a sincronia na época de maturação da espécie entre lagoas da planície de inundação do rio Cuiabá.

\section{MATERIAL E MÉTODOS}

O Pantanal é uma planície inundável de aproximadamente $137.000 \mathrm{~km}^{2}$. A alternância da estação chuvosa e seca define o clima como de caráter estacional (Hamilton et al. 1996). O regime hidrológico da região pode ser divido em quatro fases: cheia (janeiro a março) estação chuvosa, em que os rios transbordam e invadem as áreas alagáveis; vazante (abril a junho) período em que as águas estão retornando ao canal principal do rio; seca (julho a setembro) período de baixa precipitação e enchente (outubro a dezembro) época em que iniciam as chuvas e o nível da água começa a subir nos rios e lagoas (DA SiLva \& PINTO-SILVA 1989).

As lagoas da planície de inundação, conhecidas regionalmente como baías, são corpos d'água de baixa profundidade e formas variadas, com diferentes níveis de conectividade com o rio Cuiabá (DA SiLva \& PINTO-SILVA 1989). De modo geral, as lagoas de planícies de inundação são caracterizadas pela alta disponibilidade de abrigo e alimento, constituindo-se em verdadeiros berçários naturais e suprindo assim, as necessidades biológicas e ecológicas das populações que habitam ou utilizam com certa periodicidade esses corpos d'água (Cunico et al. 2002).

A região estudada está localizada na bacia do rio Cuiabá que drena uma área de aproximadamente $20.000 \mathrm{~km}^{2}$, a $145 \mathrm{~km}$ de Cuiabá, Mato Grosso, na Reserva Particular do Patrimônio Natural do SESC (RPPN SESC Pantanal), entre os meridianos $56^{\circ}$ e $57^{\circ} \mathrm{W}$ e paralelos $16^{\circ}$ e $17^{\circ} \mathrm{S}$. A área de estudo é rica em canais secundários, regionalmente chamados de corixos, com profundidade variada e conectados diretamente ao canal principal do rio. Estes funcionam como conectores entre o canal do rio e as lagoas (DA Silva \& PinTo-Silva 1989). As lagoas amostradas possuem tamanhos variados e algumas são totalmente cobertas por macrófitas aquáticas, habitats ocupados por diversas espécies de peixes de pequeno porte entre elas M. sanctaefilomenae.

Os peixes foram coletados trimestralmente em 15 lagoas no período de junho (vazante), setembro (seca) e dezembro (enchente) de 2005 e, março (cheia) de 2006. As campanhas tinham duração de seis a oito dias. As coletas foram realizadas somente na zona litorânea com cobertura de macrófitas aquáticas.

Os peixes foram coletados com uma peneira de $1 \mathrm{~m}^{3}$, confeccionada com tela de nylon de 1,5 $\mathrm{mm}$ de abertura de malha, sendo efetuados nove lances por lagoa. Os peixes capturados foram fixados em formol a 10\%, preservados em álcool a 70\% e identificados de acordo com BRITSKI et al. (1999). Medidas de comprimento furcal em milímetros foram tomadas com auxilio de um paquímetro digital.

Foram amostrados 212 indivíduos de $M$. sanctaefilomenae, distribuídos entre as 15 lagoas, sendo 99 exemplares em junho, 85 em setembro, 28 em dezembro. As gônadas foram retiradas para análise histológica. Para processamento das gônadas foi utilizada a técnica convencional para histologia com inclusão em resina de Metacrylato Glicol (Hayat 1993). Os cortes foram feitos com $5 \mu \mathrm{m}$ de espessura, corados pelo azul de toluidina (1\%). A partir da análise dos cortes em microscópio óptico, os indivíduos foram separados por sexo e estádio de maturação gonadal.

O comprimento médio de machos e fêmeas foi comparado entre períodos e no total através do teste $t$ de Student (SOKAL 
\& Rohlf 1995). Na descrição de diferenças na proporção sexual do total amostrado e entre os períodos foi utilizado o Teste do Qui-quadrado $\left(\chi^{2}\right)$ (SoKal \& RoHlF 1995).

O estádio de maturação gonadal foi estabelecido de acordo com a classificação proposta por VAzzoLER (1996): I (imaturo), II (maturação), III (maturo), IV(esvaziado) e V (repouso). De cada fêmea foi medida a maior largura de 10 folículos ovarianos através do programa MOTIC ${ }^{\circledR}$ 3.0. A largura média dos folículos ovarianos foi comparada entre períodos através da análise de variância, considerando todas as lagoas em conjunto. A distribuição de freqüência de largura de folículos ovarianos foi agrupada em classes com intervalo de $20 \mu \mathrm{m}$. A freqüência relativa das classes de largura dos folículos foi calculada em cada período e lagoa para estimar a época de maturação gonadal e se ocorria variação na fase do desenvolvimento ovocitário entre as lagoas.

A distribuição da freqüência relativa de classe de largura dos folículos ovarianos nos períodos amostrados foi utilizada para estabelecer como as fêmeas liberam os ovócitos maduros dentro de um período reprodutivo. A análise da freqüência relativa da largura do ovócito reflete sua fase de desenvolvimento. Deste modo, neste estudo foi identificado o tipo de desova segundo VAzzoler (1996): desova única; desova total; desova parcelada; desova intermitente ou sem sincronia.

O comprimento da menor fêmea com ovócitos vitelogênicos (maduro) foi utilizado para estabelecer o comprimento de primeira maturação (Cmat) por lagoa no mês de dezembro, período em que foi observado fêmeas em processo de maturação. Amostrou-se 15 lagoas, porém fêmeas maduras foram coletadas em apenas seis lagoas, uma em junho e cinco em dezembro.

O comprimento médio de primeira maturação (Cmat) considerando todas as fêmeas em conjunto foi obtido através da regressão logística: $\mathrm{p}=1 / 1+\mathrm{e}^{\alpha(\text { Cmat }-\mathrm{C})}$, onde $\mathrm{p}=$ proporção (número de maduro/número total de fêmeas); $\alpha=$ Intercepto da relação; Cmat = comprimento de primeira maturação; $\mathrm{C}=$ comprimento furcal. Os parâmetros $\alpha$ e Cmat foram estimados por regressão não linear. Esta análise estima o Cmat e seu intervalo de confiança permitindo assim a comparação entre lagoas.

O comprimento assintótico $\left(\mathrm{C}_{\infty}\right)$ foi estimado para fêmeas por local, considerando que, teoricamente, o maior peixe na amostra tem 95\% do comprimento assintótico (Pauly 1979): $\mathrm{C}_{\infty}=$ Cmáx/0,95, onde: $\mathrm{C}_{\infty}=$ comprimento assintótico, Cmáx = comprimento máximo na amostra. A relação entre o comprimento de primeira maturação (Cmat) e o comprimento máximo teórico $\left(\mathrm{C}_{\infty}\right)$ foi avaliada através de regressão linear simples.

\section{RESULTADOS}

Um total de 212 indivíduos de Moenkhausia sanctaefilomenae foi analisado, sendo 108 fêmeas e 104 machos. O comprimento furcal das fêmeas variou de 31,4 a 40,6 mm e dos machos de 29,8 a $37,9 \mathrm{~mm}$. As fêmeas foram maiores do que os machos em junho $\left(\mathrm{t}_{0,5 ; 97}=3,47 ; \mathrm{p}=0,001\right)$, em setembro $\left(\mathrm{t}_{0,5 ; 83}=5,19 ; \mathrm{p}<0,001\right)$, em dezembro $\left(\mathrm{t}_{0,5 ; 26}=3,77 ; \mathrm{p}=0,001\right)$ e considerando o total amostrado $\left(\mathrm{t}_{0,5 ; 210}=6,52 ; \mathrm{p}<0,001\right)$.

Revista Brasileira de Zoologia 25 (1): 20-27, March, 2008
Não houve diferença significativa $\left(\chi^{2}=0,52 ; p=0,47\right)$ entre o número de fêmeas (108) e machos (104) em todo o período de coleta. Porém, ocorreram diferenças significativas na proporção sexual no mês de junho $\left(\chi^{2}=8,49 ; \mathrm{p}=0,004\right)$, quando predominaram os machos (59) em relação as fêmeas (40), e setembro $\left(\chi^{2}=8,50 ; p<0,001\right)$, quando as fêmeas (58) foram numericamente superiores aos machos (27). Em dezembro foram amostradas 10 fêmeas e 18 machos não havendo diferença significativa na proporção sexual $\left(\chi^{2}=2,286 ; \mathrm{p}=\right.$ 0,131) (Fig. 1). O período de março não foi analisado, pois nenhum indivíduo foi capturado.

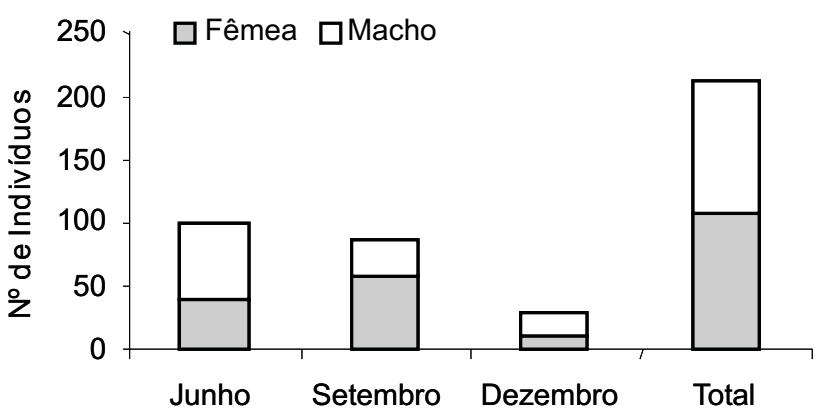

Figura 1. Proporção sexual em cada mês de coleta e todo o período para M. sanctaefilomenae, em lagoas da planície de inundação do rio Cuiabá, de junho a dezembro 2005.

Foram analisadas 40 fêmeas em junho, 58 em setembro e 10 em dezembro (Figs 2-4). Em junho, os ovócitos estavam imaturos. Em setembro, alguns folículos encontravam-se imaturos e outros em maturação. Em dezembro, as fêmeas apresentaram folículos imaturos, em maturação e maturos (Fig. 5). A maior freqüência de folículos maturos ocorreu em dezembro. Além disso, a largura média dos folículos variou significativamente entre os meses $\left(\mathrm{F}_{2 ; 903}=288,49 ; \mathrm{p}<0,001\right)$. No mês de junho, foi observada a menor largura de folículo $(21,59 \mu \mathrm{m})$ e em dezembro a maior largura $(585,21 \mu \mathrm{m})$. Esta evidencia sugere que a desova ocorreu, provavelmente, no final de dezembro ou início de janeiro. Porém, não foram observadas fêmeas com gônadas esvaziadas ou em repouso.

No mês de junho e setembro a freqüência da largura dos folículos ovarianos revelou um padrão unimodal de distribuição, em dezembro obsevou-se um padrão polimodal em todas as lagoas (Figs 6 e 7). Deste modo, sugere-se que o desenvolvimento ovocitário ocorreu sincronicamente entre as lagoas estudadas, com período de maturação semelhante.

A distribuição da largura dos folículos ovarianos no mês de junho se concentrou nas classes menores, apesar da observação de uma fêmea madura neste período. Em setembro, ocorreu um deslocamento da distribuição da largura dos folículos para larguras intermediárias e em dezembro, observa-se que a 


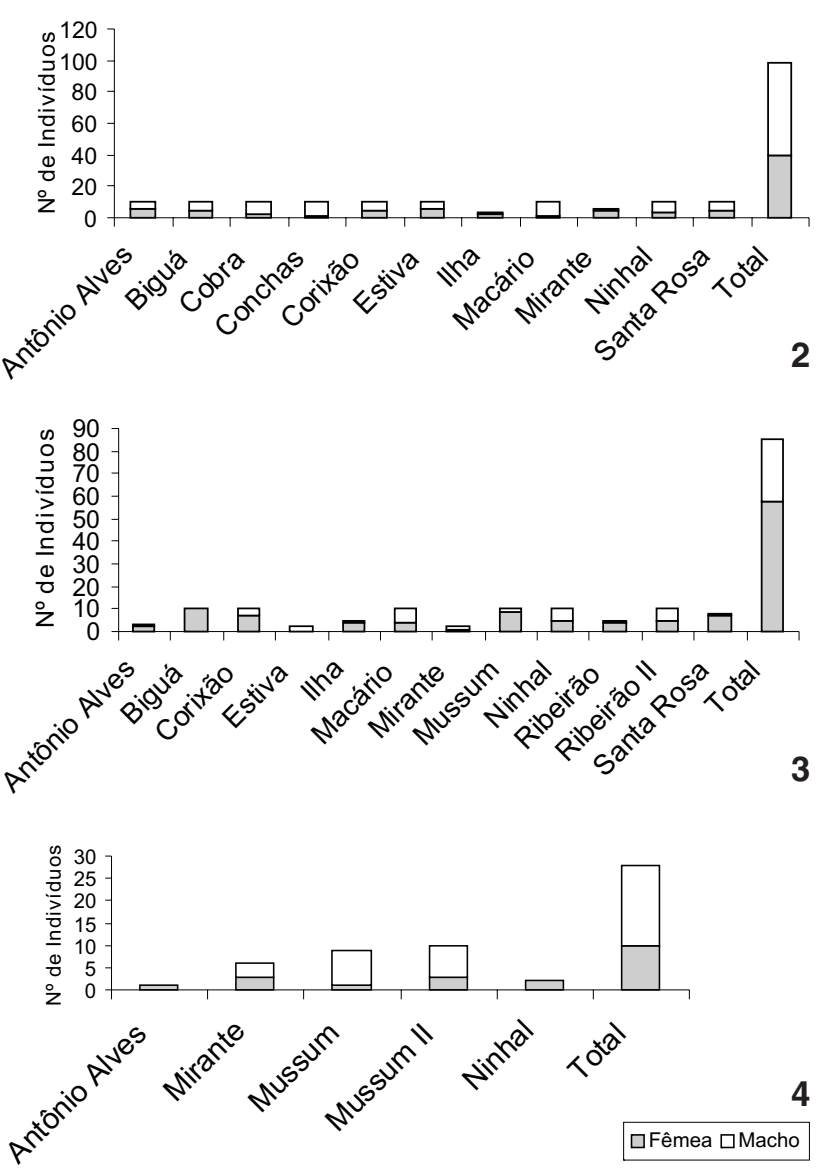

Figuras 2-4. Proporção sexual de $M$. sanctaefilomenae, em lagoas da planície de inundação do rio Cuiabá, no período de junho (3), setembro (4) e dezembro (5) de 2005.

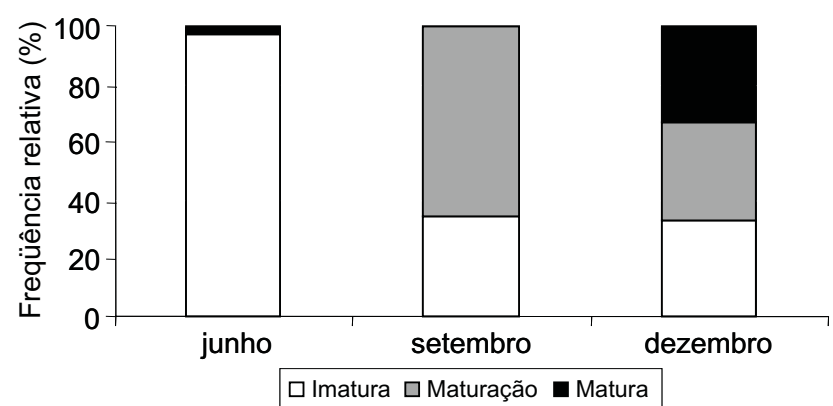

Figura 5. Freqüência relativa (\%) dos estádios de maturação gonadal de fêmeas de $M$. sanctaefilomenae, de lagoas da planície de inundação do rio Cuiabá, de junho a dezembro 2005.

freqüência relativa da largura dos folículos distribui-se tanto em larguras menores quanto intermediárias e máximas (Figs 6 e 7). Assim, a análise da distribuição de freqüência da largura dos folículos ovarianos sugere que a desova de M. sanctaefilomenae é

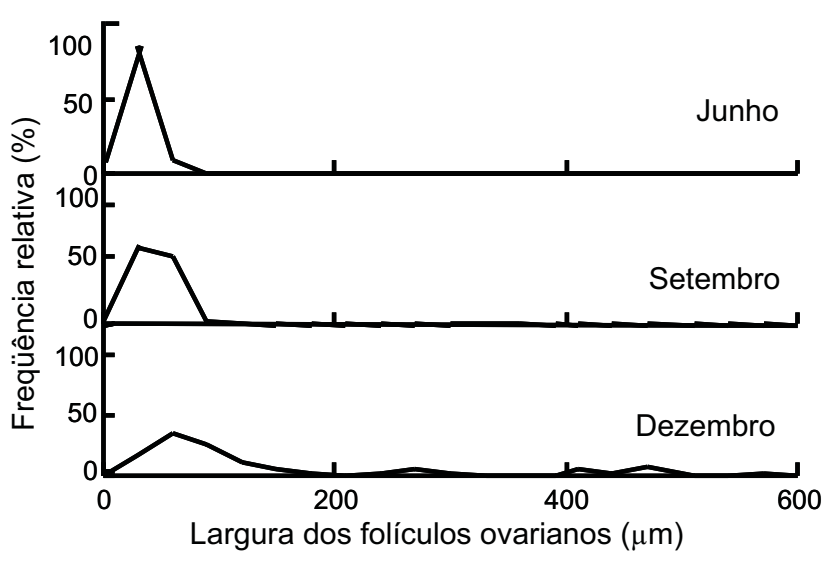

Figura 6. Distribuição de freqüência relativa (\%) da largura dos folículos ovarianos $(\mu \mathrm{m})$ de $M$. sanctaefilomenae, de lagoas da planície de inundação do rio Cuiabá, de junho a dezembro 2005.

parcelada, pois se observou um padrão polimodal na época em que foram coletadas fêmeas maduras.

O comprimento de primeira maturação (Cmat) foi estimado em 33,9 mm com intervalo de confiança entre 32,8 à $36,9 \mathrm{~mm}\left(\alpha=0,31 ; \mathrm{R}^{2}=0,66\right)$, considerando todas as fêmeas capturadas. Em duas lagoas as fêmeas apresentaram valores de comprimento de primeira maturação fora do intervalo de confiança estimado (Tab. I). O maior comprimento máximo teórico estimado foi $42,8 \mathrm{~mm}$ e o menor $36,2 \mathrm{~mm}$ (Tab. I). Como esperado, o comprimento de primeira maturação observado em

Tabela I. Comprimento de primeira maturação (Cmat), Comprimento máximo (Cmáx) e comprimento assintótico $\left(\mathrm{C}_{\infty}\right)$ de fêmeas de $M$. sanctaefilomenae, em lagoas da planície de inundação do rio Cuiabá, junho a dezembro de 2005.

\begin{tabular}{lccc}
\hline \multicolumn{1}{c}{ Lagoas } & Cmat $(\mathrm{mm})$ & $\mathrm{Cmáx}$ & $\mathrm{C}_{\infty}$ \\
\hline Antônio Alves & 36,4 & 39,2 & 41,2 \\
Estiva & 31,4 & 36,1 & 38,0 \\
Mirante & 37,9 & 40,6 & 42,8 \\
Mussum & 34,7 & 38,7 & 40,7 \\
Mussum II & 34,1 & 36,3 & 38,2 \\
Ninhal & 34,5 & 36,8 & 38,7 \\
Biguá & & 36,9 & 38,9 \\
Cobra & & 36,5 & 38,5 \\
Conchas & & 34,4 & 36,2 \\
Corixão & & 36,6 & 38,5 \\
Ilha & & 37,0 & 38,9 \\
Macário & & 36,3 & 38,2 \\
Ribeirão & & 35,2 & 37,0 \\
Ribeirão II & & 37,8 & 41,8 \\
Santa Rosa & & 38,7 & 40,7 \\
\hline
\end{tabular}

Revista Brasileira de Zoologia 25 (1): 20-27, March, 2008 


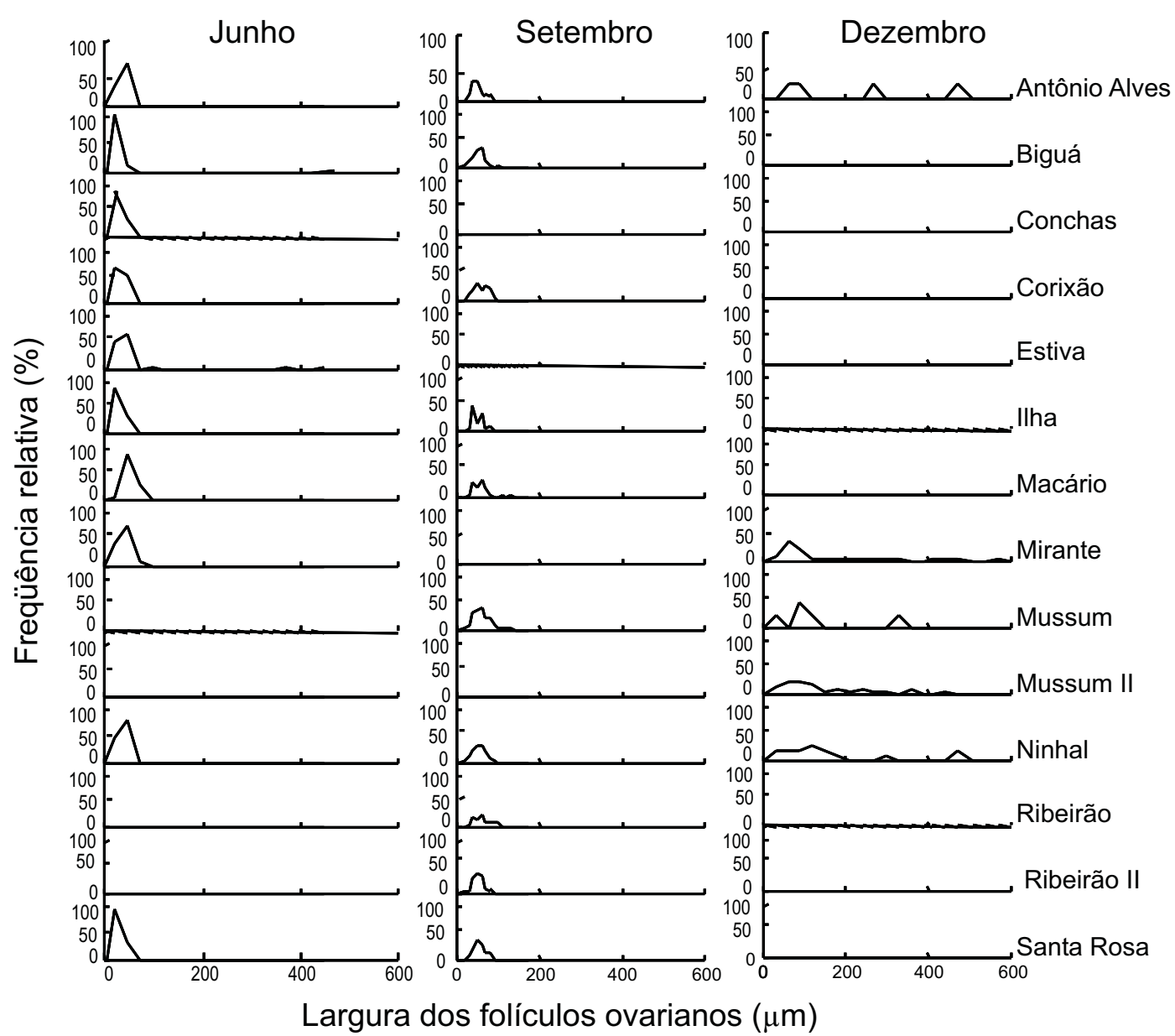

Figura 7. Distribuição de freqüência relativa (\%) da largura dos folículos ovarianos ( $\mu \mathrm{m})$ de $M$. sanctaefilomenae, de lagoas da planície de inundação do rio Cuiabá, junho 2005 á março de 2006.

cada lagoa apresentou relação positiva $\left(r^{2}=0,68 ; p=0,04\right)$ com o comprimento assintótico (Fig. 8).

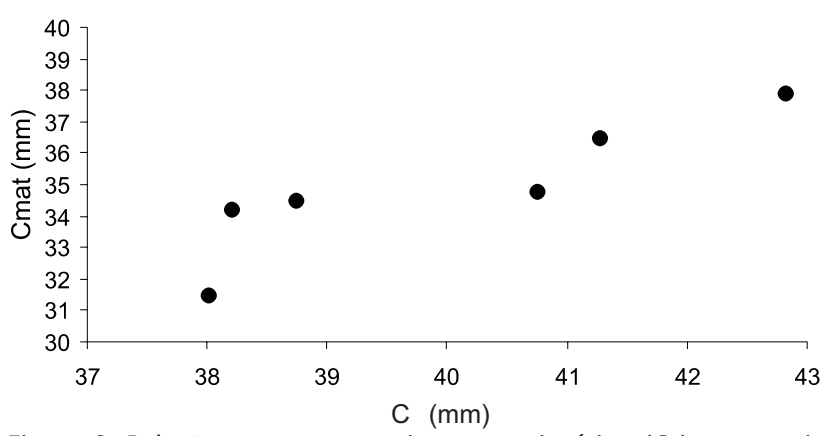

Figura 8. Relação entre o comprimento assintótico $\left(C_{\infty}\right)$ e comprimento de primeira maturação (Cmat) de fêmeas de $M$. sanctaefilomenae, de lagoas da planície de inundação do Rio Cuiabá.

\section{DISCUSSÃO}

A população de M. sanctaefilomenae não apresentou diferenças significativas na razão sexual, considerando todo período de coleta. Vicentini \& ARAúJo (2003) também não encontraram variação na proporção sexual para a população de Micropogonias furnieri (Demarest, 1823) na lagoa da Sepetiba, Rio de Janeiro.

Quando a proporção esperada não ocorre, fatores como predação e variação nas condições ambientais locais podem estar envolvidos (Mazzoni \& Caramaschi 1995, Raposo \& Gurgel 2001, GARCiA et al. 2004). As diferenças na proporção sexual podem ser atribuídas a elevada taxa de mortalidade de fêmeas como resultado da sucetibilidade a predação (MAzzoni \& CARAMASChi 1995). O tamanho, a coloração e a atividade natatória dos machos de gupies Poecilia reticulata (Peters, 1859) aumentam a probabilidade destes serem predados (RodD \& RezNICK 1997). A proporção sexual pode ser desequilibrada por causa da elevada taxa de natalidade de indivíduos de um deter- 
minado sexo (RAposo \& GuRgel 2001). O desequilíbrio da proporção sexual ainda pode ser explicado pela mortalidade específica de tamanho de um dos sexos (GARCiA et al. 2004).

No lago New Volta em Ghana, as divergências na proporção sexual nas populações de Pellonula afzeliusi (Boulenger, 1916), Cynothrissa mento (Regon, 1917), Physailia pellucida (Boulenger, 1901) e Barilius niloticus (Joanes, 1835), ocorreram devido ao aumento da mortalidade dos machos associado a segregação dos sexos quando maduros, em relação a preferência do habitat, o que tornou um dos sexo mais vulnerável à captura (REYNOLDS 1974).

Em riachos de cabeceira, a variação temporal na proporção sexual em populações de Poecilia reticulata não apresentou relação significativa com a drenagem do riacho, predação e qualidade da água (Pettersson et al. 2004). A atividade natatória de cada um dos sexos foi a explicação alternativa encontrada pelos autores para a variação da proporção sexual temporal. Em áreas temperadas, a temperatura influenciou a proporção sexual da população de genêro Gobitis (La Cepéde, 1800). Em locais com temperatura entre $14-18^{\circ} \mathrm{C}$, os machos foram mais abundantes. Quando a temperatura mudou para $20-25^{\circ} \mathrm{C}$ as fêmeas foram mais freqüentes nas amostras (Bohlena \& RitTerbusch 2000).

Diante do exposto, a principal explicação para diferenças na proporção sexual da espécie estudada pode ser a mortalidade diferencial entre machos e fêmeas. Sendo a mortalidade afetada por um único fator ou vários, ou seja, a predação, ou predação associada às características físico-química da lagoa. Portanto, a variação na proporção sexual no mês de junho e setembro pode ser resultado da mortalidade diferenciada entre fêmeas e machos, pois, os machos são menores que as fêmeas podendo estar mais suscetíveis a predação, ou pode ser resultado do efeito das variáveis ambientais associado com a habilidade de cada um dos sexos em nadar e encontrar locais mais favoráveis nas lagoas.

As fêmeas de $M$. sanctafilomenae apresentaram gônadas maturas em dezembro, quando as chuvas iniciaram, o que promove mudanças nas características do habitat. Tais como o aumento da profundidade e a disponibilização de novos ambientes na planície de inundação (HOFMANN \& Fischer 2001). Nos trópicos, as espécies de peixes regulam seu período reprodutivo para a fase de maior mudança nos habitats aquáticos. As chuvas locais e o aumento do nível da água, também estão relacionados a época de maturação de muitas espécies nas regiões tropicais (ANDRADE \& BRAGA 2005).

As populações de peixes que habitam um ambiente lótico em Volta Grande na bacia do alto rio Paraná apresentaram relação significativa da freqüência relativa das gônadas maduras com a pluviosidade (ANDRAde \& BRAga 2005). Gurgel (2004) também observou um aumento do índice gonadossomático da população de Axtyanax fasciatus (Cuvier, 1819) com o aumento do índice pluviométrico.

A época de maturação gonadal difere em populações da mesma espécie que habitam ambientes com características físi- cas e hidrológicas distintas. No rio Ubatiba, as fêmeas de Geophagus brasiliensis (Quoy \& Gaimard, 1824) apresentaram gônadas maturas ao longo de todo o ano, já as fêmeas no lago Márica apresentaram gônadas maturas entre janeiro e abril, agosto e dezembro (Mazzoni \& Iglesias-Rios 2002). Em riacho de cabeceira, como o ribeirão das Marrecas, bacia do rio Tibagi, a análise da freqüência relativa dos estádios de maturação para Axtyanax scrabipinnis paranae (Eigenmann, 1914) indicou que as fêmeas possuem gônadas maduras entre abril e agosto (VEREgUE \& ORSI 2003). A época de maturação desta espécie parece estar adaptada ao tipo de ambiente instavél que a espécie habita.

Por outro lado, AlKINS-Koo (2000) desconsidera a condição do habitat como fator determinante do período de maturação. Para a autora, a época de maturação de Gasteropelecus sternicla (Linnaeus, 1758), Corynopoma riise (Gill, 1858), Astyanax bimaculatus (Linneus, 1758), Hemigrammus unilineatus (Gill, 1858), Corydoras aeneus (Gill, 1858), e Poecilia reticulata poderia estar relacionada as características biológicas intrínsecas como comprimento corporal e filogenia.

As espécies de pequeno porte consideradas oportunistas habitam ambientes com freqüentes distúrbios, apresentando repetitivos episódios reprodutivos associados a adaptação e alta capacidade de recolonizar habitats com distúrbios (WINEMILLER \& Donald 1989). Assim, o evento reprodutivo vai ocorrer em períodos em que a condição do habitat esteja mais favorável para estas populações. Em relação a M. sanctaefilomenae na planície de inundação do rio Cuiabá a época de reprodução é sincrônica entre as lagoas e coincide com o aumento das chuvas e elevação do nível das águas. Assim, pode-se inferir que a maturação em conjunto esteja relacionada as condições semelhantes entre os habitats.

A desova de M. sanctaefilomenae em lagoas do rio Cuiabá foi parcelada, considerando que fêmeas maduras só foram encontradas com freqüência em dezembro e que neste período as fêmeas apresentam concomitantemente em suas gônadas folículos imaturos, em maturação e maturos.

Em riachos de cabeceira da bacia do alto rio Paraná, a freqüência de ocorrência dos estádios de maturação gonadal, assim como a distribuição da freqüência relativa dos diâmetros dos ovócitos de gônadas maduras da população de Aspidoras fuscoguttatus (Nijssen \& Isbrucker, 1976), sugerem desova parcelada durante todo ano (Araujo \& GuarutTi 2002), pois as fêmeas apresentaram gônadas imaturas, em maturação, matura e esgotada em todos os meses de coleta. No reservatório Itumbiara, Goiás, Hojo et al. (2004) evidenciaram desova parcelada para Moenkhausia intermédia (Eigenmem, 1908) com eventos reprodutivos o ano todo e fêmeas que apresentavam gônadas em todos os estádios de maturação gonadal.

A desova parcelada é freqüente em espécies de pequeno porte, todavia alguns trabalhos têm evidenciado a desova total. A população de Axtyanax fasciatus apresentou desova total no rio Ceará Mirim, Rio Grande do Norte (GURGel 2004), assim como a população de Geophagus brasiliensis apresentou sincronia 
no desenvolvimento ovócitário, o que sugere desova única no rio Ubatiba e no lago Maricá (Mazzoni \& Iglesias-Rios 2002).

O comprimento médio de primeira maturação definido como o comprimento no qual 50\% dos indivíduos da população apresentam gônadas em maturação (BEVERTON 1992), para M. sanctaefilomenae foi de $33,9 \mathrm{~mm}$. Com exceção de duas lagoas, o comprimento de primeira maturação das fêmeas de $M$. sanctaefilomenae estava compreendido dentro do intervalo de confiança estimado. O comprimento de primeira maturação pode apresentar diferenças entre populações da mesma espécie em ambientes com características físicas e regime hidrológico distintos. No rio Ubatiba, a população de Geophagus brasiliensis apresentou comprimento de primeira maturação foi de $5,9 \mathrm{~cm}$ e no lago Márica foi 9,5 cm (MAzzoni \& IgLEsias-Rios 2002). TARKAN (2006) comparou o comprimento de primeira maturação de duas espécies de ciprinídeos Rutilus rutilus (Linneus, 1758) e Scardinius erythrophthalmus (Linneus, 1858) de diferentes regiões, e evidenciou que ocorrem mudanças no comprimento de maturação em resposta as variações dos fatores ambientais.

Na literatura inexistem trabalhos com estimativa do comprimento máximo teórico para M. sanctaefilomenae, mas segundo BRITSKi et al. (1999) a espécie pode alcançar cerca de $70 \mathrm{~mm}$, valor este bem acima do encontrado no presente trabalho $(42,8$ $\mathrm{mm})$. Na planície de inundação da bacia do rio Paraná, a elevada taxa de crescimento observado nas espécies de peixes que são presas ocorre porque quanto mais rápido elas atingirem o comprimento máximo maiores serão as chances de sobrevivência em locais com alta pressão de predação (LizAma \& AMBrósıo 2003). Quando a mortalidade específica de tamanho ocorre os individuos de maiores comprimentos podem ser favorecidos (TreXLer et al. 1992).

Moenkhausia sanctaefilomenae apresentou relação positiva entre o comprimento máximo teórico e comprimento de primeira maturação, confirmando que estes parâmetros co-variam. STAMPs et al. (1998) também encontraram uma relação positiva entre estes dois parâmetros em estudos comparativos. Assim, essa relação ocorre porque os indivíduos devem atingir um tamanho específico de corpo antes de serem capazes de reproduzir (KING 1995).

\section{AGRADECIMENTOS}

À CAPES pela concessão de bolsa de mestrado à primeira autora. Ao Centro de Pesquisa do Pantanal (CPP/MCT) e PELD/ CNPq site - 12 pelo apoio financeiro. À Estância Ecológica SESC Pantanal pelo apoio logístico nos trabalhos de campo.

\section{LITERATURA CITADA}

Alkins-Koo, M. 2000. Reproductive timing of fishes in a tropical intermittent stream. Environmental Biology of Fishes 57: 49-66.

Andrade, P.M. \& F.M.S. Braga. 2005. Reproduction seasonality of fish from a lotic strech of the grand river, high Paraná basin, Brazil. Brazilian Journal of Biology 63 (3): 387-394. Araújo, B.R. \& V. Garutti. 2002. Biologia Reprodutiva de Aspidoras fuscoguttatus (Siluriformes, Callichthyidae) em riachos de cabeceira da bacia do alto rio Paraná. Iheringia, Série Zoologia, 92 (4): 88-98.

Beverton, R.J.H. 1992. Patterns of reproduction strategy parameters in some teleost fishes. Jounal of Fish Biology 41: 137-160.

Bohlena, J. \& D. RitTerbusch. 2000. Which factors affect sex ratio of spined loach (genus Cobitis) in Lake Muggelsee? Environmental Biology of Fishes 59: 347-352.

Britisk, H.A.; K.Z. Silimon \& B.S. Lopes. 1999. Peixes do pantanal manual de identificação. Brasília, Embrapa, 184p.

Casatti, L.; F. Langeani \& R.M. Castro. 2001. Peixes de Riacho do Parque Estadual Morro do Diabo, Bacia do Alto Rio Paraná. Biota Neotropical 1 (1): 1-15.

Cunico, A.M.; W.J. Graça; S. Veríssimo \& L. Mauricio. 2002. Influência do nível hidrológico sobre a assembléia de peixes em lagoa sazonalmente isolada da planície de inundação do alto rio Paraná. Acta Scientiarum 24 (2): 383-389.

DA SiLVA, C.J. \& V. PINTo. 1989. Macrófitas aquáticas e as condições físicas e químicas dos alagados, corixos, e rios ao longo da rodovia Transpantaneira - Pantanal Matogrossense (Poconé, MT). Revista Brasileira de Biologia 49 (3): 691-697.

Garcia, A.M.; J.P. Vieira; K.O. Winemiller \& M.B. Raseira. 2004. Reproductive cicle and spationaltemporal variation in abundance of the one-sided livebear Jenynsia multidentada, in Patos Lagos, Brazil. Hydrobiologia 515: 39-48.

Gurgel, H.C.B. 2004. Estrutura populacional e época de reprodução de Astyanax fasciatus (Cuvier) (Chacidae, Tetragonopterinae) do Rio Céara Mirim, Poço Branco, Rio Grande do Norte, Brasil. Revista Brasileira de Zoologia 21 (1): 131-135.

Hamilton, S.K.; S.J. Sippel \& J.M. Melack. 1996. Inundation patterns in the Pantanal of South America determined from passive microwave remote sensing. Archiv Fur Hydrobiologie 137 (1): $1-23$.

Hayat, M.A. 1993. Stains and Cythochemical methods M. A. Hayat. Includes biographical references and index Stains and staining (microscope). 2. Electron microscopyTechonique. New York, Plenum Press, 445p.

Hofmann, N. \& P. Fischer. 2001. Seasonal changes in abundance and age structure of burbot Lota lota (L.) and stone loach Berbatula barbatula (L.) in the littoral zone of a pre-alpine lake. Ecology of Freshwater Fish 10: 21-25.

Hojo, S.E.R.; G.B. Santo \& N. Bazzoli. 2004. Reproductive biology of Moenkhausia intermedia (Eigenmann) (Pisces, Characiformes) in Itumbiara Reservoir, Goiás, Brazil. Revista Brasileira de Zoologia 21 (3): 519-524.

KING, M.G. 1995. Fisheries biology: assessment and management. Oxford, Fishing News Book Blackwell Science, 341p. Kramer, D.L. 1978. Reproduction Seasonality in Fishes of a Tropical Stream. Ecology 59 (5): 978-985.

Lizama, M.A.P. \& A.M. Ambrósıo. 1999. Relação peso-comprimen-

Revista Brasileira de Zoologia 25 (1): 20-27, March, 2008 
to e estrutura de nove espécies de peixes da família Characidae na planície de inundação do alto rio Paraná, Brasil. Revista Brasileira Zoologia 16 (3): 779-788.

Lizama, M.A.P \& A.M. Ambrósio. 2002. Condition factor in nine species of fish of the Characidae family in the high Paraná River floodplain, Brazil. Revista Brasileira de Biologia 62 (1): 113-124.

Lizama, M.A.P. \& A.M. Ambrósio. 2003. Crescimento, recrutamento e mortalidade do pequi Moenkhausia intermedia (Osteichthyes, Characidae) na planície de inundação do alto rio Paraná, Brasil. Acta Scientiarum 25 (2): 328-333.

Lowe-McConnell, R.H. 1999. Estudos ecológicos de comunidades de peixes tropicais. São Paulo, EDUSP, 584p.

Machado-Allison, A. 1987. Los Peces de los Ilanos de Venezuela: un ensayo sobre su história natural. Caracas, Universidad Central de Venezuela, Consejo de desarollo Científico y Humanístico, 144p.

Mazzoni, R. \& E.P. Caramaschi. 1995. Size structure, sex ratio and onset of sexual maturity of two species of Hypostomos. Journal of Fish Biology 47: 841-849.

Mazzoni, R. \& R. Iglesisas-Rio. 2002. Environmentally related life history variations in Geophagus brasiliensis. Journal of Fish Biology 61: 1606-1618.

Pauly, D. 1979. Teory and management of tropical multispecies stocks. Manila, ICLARM Studies and Reviews 1, 35p.

Pettersson, L.B.; I.W. Ramnarine; S.A. Becher; R. Mahabir \& A.E. MagurRan. 2004. Sex ratio dynamics and fluctuating selection pressures in natural population of Trinidadian guppy, Poecilia reticulata. Behavior Ecology Sociobiology 55: 461-468.

Raposo, R.M.G. \& H.C.B. GuRgel. 2001. Estrutura populacional de Serrasalmus splilopeura Kner, 1860 (Pisces, Serrasalmidae), da lagoa de Extremoz, Estado do rio Grande do Sul. Revista Brasileira de Zoologia 21 (1): 131-135.

Reis, R.E.; O. Kullander \& C.J. Ferraris-Jr. 2003. Check list of the freshwater fishes of South and Central America. Porto Alegre, EDIPUCRS, 742p.

Reynolds, J.D. 1974?. Biology of the Small Pelagic Fishes in the New Volta Lake in Ghana. Part III: Sex and Reproduction. Hydrobiologia 45 (4): 489-508.
Roff, D.A. 1984. The evolution of immigration and some life history parameters in marine fish. Environmental Fish Biology 22: 133-146.

Rood, F.H. \& D.N. Rezcnick. 1997. Variation in the demography of guppy population: the importance of predation and life histories. Ecology 78 (2): 405-418.

Silvano, J.; C.L.C. Oliveira; C.B. Fialho \& H.C.B. Gurgel. 2003. Reproductive period and fecundity of Serrapinus piaba (Characidae: Cheirodontidae) from the rio Ceará Mirim, Rio Grande do Norte, Brasil. Neotropical Ichthyology 1 (1): 61-66.

SoKaL, R.R. \& F.J. RoHLf. 1995. Biometry. New York, W.H. Freeman, $3^{\text {rd }}$ ed., 887p.

Stamps, J.A.; M.P. Mangel \& J.A. Phillips. 1998. A new look at relationships between size at Maturity and asymptotic size. The American Naturalist 152 (3): 470-479.

TARKAN, A.S. 2006. Reproductive ecology of two cyprinid fishes in an oligotrophic lake near the southern limits of their distribution range. Ecology of Freshwater Fish 15: 131-138.

Trexler, J.C.; J. Travis \& M. Mcmanus. 1992. Effects of habitat and body size on mortality rates Poecilia latipinna. Ecology 73 (6): 2224-2236.

VAzzolER, A.E.A.M. 1996. Biologia da reprodução de peixes teleósteos: teoria e prática. Maringá, EDUEM, 169p.

Veregue, A.M. \& M.L.Orsi. 2003. Biologia reprodutiva de Astianax scabripinnis paranae (Eigenmem) (Ostheichthyes, Characidae), do ribeirão das Marrecas, bacia do rio Tibagi, Paraná. Revista Brasileira de Zoologia 20 (1): 97-105.

Vincentini, R.N. \& F.G. Araújo. 2003. Sex ratio size structure of Micropogonias furnieri (Desmarest, 1823) (Perciformes, Sciaenidae) in Sepetiba bay, Rio de Janeiro, Brasil. Journal of Biology 63 (4): 559-566.

Winemiller, K.O. 2005. Live History, Population Regulation, and implication for fisheries management. Canada Journal Fisheries Aquatic Science 62: 872-885.

Winemiller, K.O. \& C. Donald. 1989. La evolucion de las estrategias de vida en los peces de los Ilanos Occidentales de Venezuela. Biollania 6: 77-122.

Wootton, R. 1998. Ecology of teleost fishes. New York, Academic Publishers, $2^{\text {nd }} e d ., 386 p$.

Received in 24.IV.2007; accepted in 22.II.2008. 